\title{
Monte Carlo studies on the well-width dependence of carrier capture time in graded-index separate confinement heterostructure quantum well laser structures
}

\author{
Yeeloy Lam ${ }^{\text {a) }}$ and Jasprit Singh \\ Solid State Electronics Laboratory, Department of Electrical Engineering and Computer Science, \\ The University of Michigan, Ann Arbor, Michigan 48109-2122
}

(Received 15 April 1993; accepted for publication 30 July 1993)

\begin{abstract}
The total carrier capture time and the quantum well width are both important parameters affecting the graded-index separate confinement heterostructure (GRINSCH) quantum well laser modulation speed limit. However, discrepancies exist in the literature on the well-width dependence of the carrier capture times. To study this phenomenon, we have developed a Monte Carlo technique to simulate carrier relaxation in GRINSCH quantum well structures. Our results show that the carrier capture time increases with the density of carrier injection. Furthermore, depending on the concentration of injected carriers, the capture time will cither decrease, remain the same, or increase with increases in the well width. At lasing conditions, the times are more or less independent of the well width up to $100 \AA$. We compare our calculations to published experiments and find good agreements.
\end{abstract}

The total carrier capture time in graded-index separate confinement heterostructure (GRINSCH) quantum well lasers has been identified as an important factor in nonlinear gain compression, ${ }^{1-3}$ a key parameter determining its ultimate direct modulation speed. Such effects have been studied previously, ${ }^{4}$ using diffusion theory and ignoring the quantum capture time. However, the calculations by Brum et al. ${ }^{5}$ predict a $10-100$ ps quantum capture time, indicating this time should not be ignored. Knowledge on how the carrier capture time changes (if at all) with the well width and the carrier injection density is crucial for optimization of the laser structure for high speed operation. While some experiments ${ }^{6,7}$ report no well-width dependency, others either observe an increasing ${ }^{8}$ or decreasing ${ }^{9}$ trend. To the best of our knowledge, no studies of the carrier relaxation process including scattering in the three-dimensional (3D) confinement layer and the two-dimensional (2D) quantum well in a complete and consistent manner have been reported to date. In this letter we present the results for varying well size and injection densities in linear GRINSCH and parabolic GRINSCH quantum well laser structures. Our theoretical investigation is based on the ensemble Monte Carlo simulation of the carrier relaxation processes in these structures. The structures studied consist of a single GaAs quantum well (40-100 $\AA$ ) sandwiched between symmetrical linearly (L-GRINSCH) or parabolically (P-GRINSCH) graded (70\%-14\% A1) AlGaAs confinement layers of thickness $2000 \AA$.

In our Monte Carlo method, we model the carrier transport in the 3D graded-index region and the 2D well region separately. The 2D well region includes the quantum well layer and a thin layer of its neighboring barrier that experiences significant quantum confinement effects. The dominant scattering mechanism in this region is the polar-optical phonon (POP) scattering. Using the Fermi Golden Rule, the intersubband POP rates are given by ${ }^{10}$

a) Present address: Nanyang Technological University, Singapore.

$$
\begin{aligned}
S_{m n}^{\mathrm{POP}}= & \frac{e^{2} \omega_{0}}{8 \pi \epsilon_{0}}\left[\frac{1}{\epsilon_{\infty}}-\frac{1}{\epsilon_{s}}\right]\left(N_{q}+\frac{1}{2} \mp \frac{1}{2}\right) \\
& \times \int \frac{H_{m n}(Q) Q}{Q^{2}+Q_{0}^{2}} \delta\left[E\left(\mathbf{k}_{2}\right)-E\left(\mathbf{k}_{1}\right) \pm \hbar \omega_{0}\right] d \mathbf{k}_{2},
\end{aligned}
$$

where $N_{q}$ is the phonon occupation number, $\epsilon_{\infty}=10.92$ and $\epsilon_{s}=12.90$. The initial and final-state wave vectors of the electron are $\mathbf{k}_{1}$ and $\mathbf{k}_{2}$, while $Q= \pm\left(\mathbf{k}_{1}-\mathbf{k}_{2}\right)$ and $q$ are the phonon wave vector components parallel and perpendicular to the well layer. For simplicity, the carrier screening effects ${ }^{11}$ are included in Eq. (1) with $Q_{0}$ $=\sqrt{e^{2} n / \epsilon K_{B} T}$ as the Debye screening wave vector. The multisubband coupling coefficients are given by

$$
\begin{aligned}
H_{m n}(Q)= & \iint d z_{1} d z_{2} F_{m n}\left(z_{1}\right) F_{m n}\left(z_{2}\right) \\
& \times \exp \left(-Q\left|z_{1}-z_{2}\right|\right),
\end{aligned}
$$

where $F_{m n}(z)=F_{m}(z) F_{n}(z)$. Employing a finite difference method, we obtain the envelope function $F_{m}(z)$ by solving the Schrödinger equation that describes the electronic states in the GRINSCH structure. For completeness, we also include the acoustic phonon (ACP) scattering rates in our model, although they are about 100 times smaller than the POP rates.

Electron-electron interactions become significant when the carrier density is moderately high. The total screened electron-electron scattering rates for the $3 \mathrm{D}$ rcgion is found, using the Fermi Golden Rule, ${ }^{12,13}$ to be

$$
\Gamma_{e-e}\left(\mathbf{k}_{0}\right)=\frac{n m^{*} e^{4}}{4 \pi \hbar^{3} \epsilon_{0}^{2} \beta^{2} N} \sum_{\text {all } k s} \frac{\left|\mathbf{k}_{0}-\mathbf{k}\right|}{\left[\beta^{2}+\left|\mathbf{k}_{0}-\mathbf{k}\right|^{2}\right.},
$$

where the sum is extended over the $N$ carriers of the $3 \mathrm{D}$ ensemble, and $\beta=0.5 Q_{0}$ is the inverse effective screening length. ${ }^{13}$ We have implemented both the $2 \mathrm{D}$ and $3 \mathrm{D}$ e-e scattering mechanisms in our model using a rejection method. ${ }^{14,15}$ 


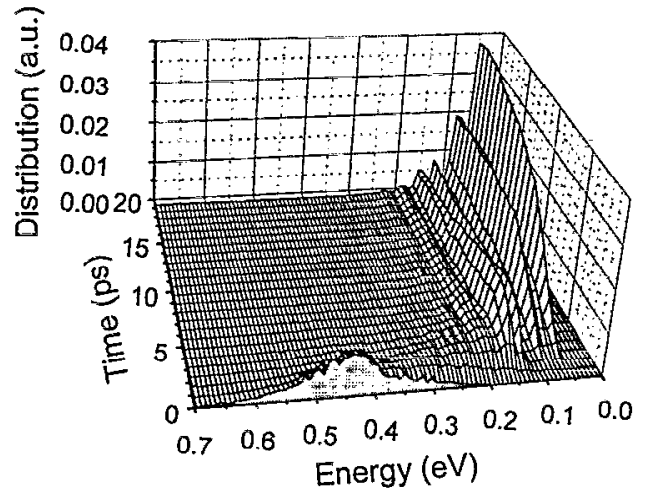

FIG. 1. Time evolution of the energy distribution for an ensemble of 10000 relaxing carriers, injected from the edge of the confinement layer with a kinetic energy of $15 \mathrm{mcV}$ at time $=0$ ps. The simulation is performed on a L-GRINSCH structure with a $50 \AA$ quantum well, under an applied electric field of $2 \mathrm{kV} / \mathrm{cm}$.

We have successfully implemented the modeling of the carrier capture and reemission processes-the $3 \mathrm{D} \leftrightarrow 2 \mathrm{D}$ transitions made by the carriers between the 3D and the 2D regions, within the Monte Carlo approach in our model. The technique is based on the reasoning that if we carry out a 2D Monte Carlo calculation for the graded region by including all subbands, then in principle the same result can be obtained from a corresponding 3D Monte Carlo calculation. In practice, we find that by considering up to five subbands, a satisfactory modeling of the $3 \mathrm{D} \leftrightarrow 2 \mathrm{D}$ transitions can be achieved. In our method, we model both the $3 \mathrm{D} \rightarrow 2 \mathrm{D}$ and $2 \mathrm{D} \rightarrow 3 \mathrm{D}$ transitions as phonon-assisted processes. A transition energy level $E_{\mathrm{tr}}$ is introduced to facilitate the $2 \mathrm{D} \leftrightarrow 3 \mathrm{D}$ transition. We carry out the $3 \mathrm{D} \rightarrow 2 \mathrm{D}$ transition, if upon the termination of a $3 \mathrm{D}$ free flight, the total carrier energy after a phonon emission scattering process is expected to fall below $E_{\mathrm{tr}}$. A final 2D state is chosen from the nearest subband considering energy and momentum conservation. We perform the reverse $2 \mathrm{D} \rightarrow 3 \mathrm{D}$ transition when the total energy of a well carrier is expected to exceed $E_{\mathrm{tr}}$ after a phonon absorption scattering process. To conserve the in-plane energy and momentum, the in-plane wave vector is unchanged. Its $z$-directed wave vector is then obtained by considering energy conservation; its $z$ orientation is randomly selected. In our study, we set $E_{\mathrm{tr}}$ equal to one $k_{B} T$ ( $26 \mathrm{meV}$ ) above the subband minimum of the highest (fifth) state being considered. This setting is confirmed with a series of simulation runs. We find essentially no change if $E_{\mathrm{tr}}$ is increased somewhat, showing that including five subband levels is quite adequate. Details of the Monte Carlo program will be presented in a longer article. ${ }^{16}$

In our calculations, we simulate how an ensemble of carriers injected at the barrier edge progress through the graded-index region, are captured by the quantum well and relax to their thermal equilibrium. Simulations of the carrier relaxation processes are carried out under different injected carrier densities; i.e., $10^{16}, 5 \times 10^{17}$, and $10^{18} \mathrm{~cm}^{-3}$, labeled as low, medium, and high injection densities, respectively. In Fig. 1, we show the time development of the

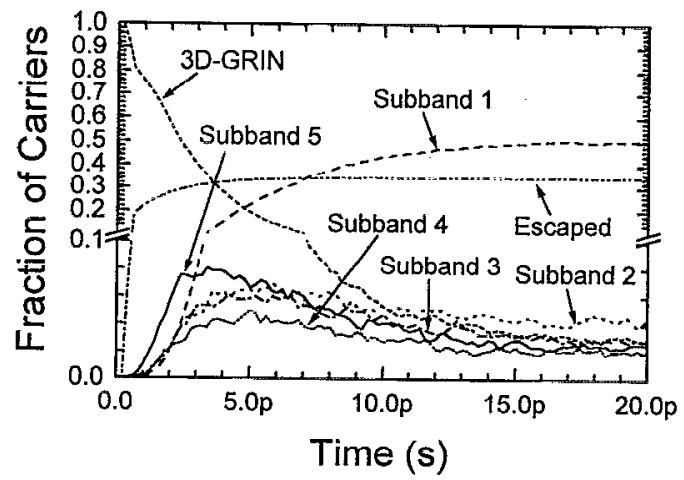

FIG. 2. The distribution of carriers in the structure for the same simulation run as in Fig. 1.

ensemble energy distribution during the simulation, under a low injection condition for the $50 \AA$ well L-GRINSCH structure. The distribution of carriers in the structure for the same simulation is shown in Fig. 2. The fraction of carriers in the "3D-GRIN" region decreases from a maximum of $100 \%$ upon injection to a low of $3 \%$ after about $15 \mathrm{ps}$, while the well-state (first subband) occupancy increases from zero to its maximum in the same period. We further note that the occupancies of the various subbands do indeed correctly portray the transport of the carriers through the subbands.

An interesting difference between the $\mathrm{L}$ - and P-GRINSCH structures lies in the carrier capture time. As shown in Fig. 3 (curves 5 and 6), the L-GRINSCH structures are significantly faster than the P-GRINSCH structures. We have also obtained good agreement between our simulation and published experiments ${ }^{7}$ - by comparing our calculated carrier capture time constants of 6.7 and $8.8 \mathrm{ps}$ (obtained from simple exponential curve fitting) and the experimental ${ }^{7}$ time constants of 8.2 and $10 \mathrm{ps,} \mathrm{for} 50 \AA$ well width L- and P-GRINSCH structures, respectively.

We also study the capture time dependence on both the well width and the carrier density. From Fig. 3 (curves, 3, 4 , and 5), we see that the carrier capture time increases with the density of carrier injection. Also, the capture time exhibits a nonmonotonical trend with the well width at low injections. Oscillatory behavior has been predicted by

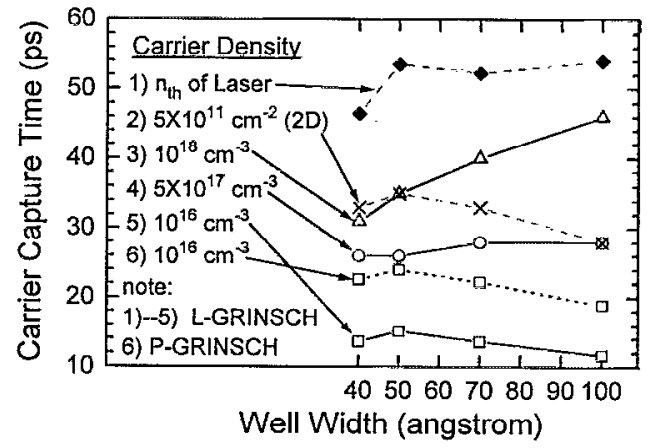

FIG. 3. Calculated carrier capture time as a function of well width and injection density. We define this time as the time needed for the well-state occupancy to reach $95 \%$ of the equilibrium value. 
TABLE I. The 2D and 3D threshold carrier density $n_{\text {th }}$ of the L-GRINSCH quantum well lasers.

\begin{tabular}{lcccl}
\hline \hline Well width $(\AA)$ & 40 & 50 & 70 & 100 \\
\hline $2 \mathrm{D} n_{\text {th }}\left(10^{12} \mathrm{~cm}^{-2}\right)$ & 1.09 & 1.10 & 1.16 & 1.30 \\
$3 \mathrm{D} n_{\text {th }}\left(10^{18} \mathrm{~cm}^{-3}\right)$ & 2.74 & 2.20 & 1.65 & 1.3 \\
\hline \hline
\end{tabular}

Brum et al..$^{5}$ At higher injections, monotonical trends appear. We can observe from Fig. 3 either decreasing, flat, or increasing capture times with the well width, depending on the injection conditions. Carrier capture times are measured under high current injection conditions by Weiss et al. ${ }^{8}$ to which reported longer carrier capture times in wider well structures. This is in agreement with our results at high injections. On the other hand, the experiments in Ref. 6 with photoluminescence and photoexcitation measurements reported no dependencies of the carrier capture times on the well width. Since moderate carrier injections are expected in these experiments, our calculations also predict no significant capture time dependency on the well width-in agreement with the experiments.

We shall now examine the well-width dependence under lasing conditions, where the threshold carrier density $n_{\text {th }}$ varies with the well sizes. Table I tabulates the calculated $n_{\text {th }}$ for the L-GRINSCH lasers having typical laser parameters. ${ }^{17}$ Curve 1 in Fig. 3 shows the calculated capture times at the threshold densities. We observe that the carrier capture times under lasing conditions are more or less independent of the well width. This behavior can be attributed to two opposing factors-changes in the $2 \mathrm{D}$ and 3D carrier densities.

For a particular 3D injection density, the 2D carrier density in the quantum well increases with the well width. Since carrier screening in the well varies with the $2 \mathrm{D}$ carrier density, an increase in the carrier capture time with the well width is expected. This behavior is confirmed by comparing curve 2 (constant 2D density) and curve 3 (constant 3D density) in Fig. 3. Under lasing conditions, the 2D $n_{\text {th }}$ increases with the well size (by $\sim 20 \%$ for 40 $\AA \rightarrow 100 \AA$ well). Hence, the capture time should also be proportionally prolonged in the wider well lasers. On the other hand, from Table I we note that the increases in the $2 \mathrm{D} n_{\text {th }}$ are accompanied by decreases in the $3 \mathrm{D} n_{\text {th }}$ (to $\sim 50 \%$ of its value when the well width is increased from $40 \AA$ to $100 \AA$ ). Faster capture time is expected from a lower 3D carrier density. If we were to consider only the effects of 3D carrier screening and assuming a square-root relationship, $a \sim 30 \%$ reduction in the capture time may be expected. However, the actual effect will be much smaller since the average carrier dwell time in the 3D graded-index region is a minor portion of the overall capture time. The trend in curve 1 indicates that this effect is less than $10 \%$.

In summary, we have successfully modeled the carrier relaxation process in GRINSCH quantum well laser structures under different injection conditions. Our calculations show the carrier capture in L-GRINSCH structures to be faster than in P-GRINSCH structures. We find that the carrier capture time is greatly increased at high injections. At low to moderate injections, the capture time may either remain insensitive or decrease with an increase in the well width; under high injections, the capture time is found to be longer in wider quantum well structures. However, under lasing conditions, the capture times are more or less independent of the well width. We compare our calculations to published experiments and find good agreements.

This work was funded by a grant from the Office of Naval Research Contact N00014-90-J-1831.

${ }^{1}$ M. Yamada and Y. Suematsu, J. Appl. Phys. 52, 2653 (1981).

${ }^{2}$ W. Sharfin, J. Schlafer, W. Rideout, B. Elman, E. Koteles, R. B. Lauer, J. LaCourse, and F. D. Crawford, presented at The Conference on Lasers and Electro-Optics, CLEO 91, May 12-17, Baltimore, MD.

${ }^{3}$ W. Rideout, W. F. Sharfin, E. S. Koteles, M. O. Vassell, and B. Elman, IEEE Photon. Tech. Lett. 3, 784 (1991).

${ }^{4}$ R. Nagarajan, T. Fukushima, S. W. Corzine, and J. E. Bowers, Appl. Phys. Lett. 59, 1835 (1991).

\$J. A. Brum and G. Bastard, Phys. Rev. B 33, 1420 (1986); J. A. Brum, T. Weil, J. Nagle, and B. Vinter, Phys. Rev. B. 34, 2381 (1986).

${ }^{6} \mathrm{~B}$. Deveaud, in Proceedings of the 20th International Conference on the Physics of Semiconductors, Thessaloniki, Greece, edited by E. M. Anastassakis and J. D. Joannopoulos (World Scientific, Singapore, 1990), p. 1201.

${ }^{7}$ S. Morin, B. Deveaud, F. Clerot, K. Fugiwara, and K. Mitsunaga, IEEE J. Quantum Electron. 27, 1669 (1991).

${ }^{8}$ We compare the carrier capture times (Table I) between samples 1 and 2 in the published work by S. Weiss, J. M. Wiesenfeld, D. S. Chemla, G. Raybon, G. Sucha, M. Wegener, G. Eisenstein, C. A. Burrus, A. G. Dentai, U. Koren, B. I. Miller, H. Temkin, R. A. Logan, and T. Tanbun-Ek, Appl. Phys. Lett. 60, 9 (1992).

${ }^{9}$ L. Davis, Y. Lam, Y.-C. Chen, S. Sethi, J. Singh, and P. Bhattacharya, presented at Electronics Materials Conference, Santa Barbara, CA, June, 1993.

${ }^{10} \mathrm{~K}$. Yokoyama and K. Hess, Phys. Rev. B 31, 6872 (1985); ibid 33, 5595 (1986).

${ }^{11}$ B. K. Ridley, Quantum Processes in Semiconductors, 2nd ed. (Oxford University Press, New York, 1988).

${ }^{12}$ C. Jacoboni and P. Lugli, The Monte Carlo Method for Semiconductor Device Simulation (Springer, New York, 1989).

${ }^{13}$ P. Lugli and D. K. Ferry, Appl. Phys. Lett. 46, 594 (1985).

${ }^{14}$ S. M. Goodnick and P. Lugli, Phys. Rev. B 37, 2578 (1988).

${ }^{15}$ R. Brunetti, C. Jacoboni, A. Matulionis, and V. Dienys, Physica B 134, 369 (1985).

${ }^{16}$ Y. Lam and J. Singh (unpublished).

${ }^{17}$ Y. Lam, J. P. Loehr, and J. Singh, IEEE J. Quantum Electron. 28, 1248 (1992). 